\title{
Caractéristiques rhéologiques et gélification thermique du concentré de protéines de lactosérum
}

\author{
P Gault, M Mahaut, J Korolczuk \\ INRA, Laboratoire de recherches de technologie laitière, \\ 65, rue de Saint-Brieuc, 35042 Rennes, France
}

(Reçu le 4 septembre 1989; accepté le 7 mars 1990)

\begin{abstract}
Résumé - Le comportement rhéologique et la cinétique de gélification thermique d'un concentré de protéines de lactosérum (CPL) diafiltré et délipidé ont été étudiés à partir d'un viscosimètre à cylindres coaxiaux. Les caractéristiques rhéologiques des gels ont été estimées avec un rhéomètre à compression. Les solutions du $\mathrm{CPL}$, contenant de 4 à $14 \%$ de protéines, à $25^{\circ} \mathrm{C}$ et à pH 7 , présentent un comportement newtonien pour des vitesses de cisaillement comprises entre 82 et $955 \mathrm{~s}^{-1}$. Le logarithme de la viscosité est une fonction linéaire de la concentration en protéines. L'énergie d'activation d'écoulement augmente linéairement avec la concentration en protéines, à partir de $6 \%$, d'environ $13 \mathrm{~kJ} \cdot \mathrm{mol}^{-1}$ pour l'eau, à $20 \mathrm{~kJ} \cdot \mathrm{mol}^{-1}$ pour le $\mathrm{CPL}$ à $14 \%$ de protéines. A $60^{\circ} \mathrm{C}$, les solutions à $\mathrm{pH} 4$ et à $10 \%$ de protéines présentent un comportement rhéofluidifiant en cours de gélification thermique. A $60^{\circ} \mathrm{C}$, l'augmentation de la viscosité est la plus rapide à pH 4 et 9 , et, entre $\mathrm{pH} 5$ et 7,5 , les solutions ne gélifient pas. $\mathrm{A} 70^{\circ} \mathrm{C}$, le CPL gélifie entre $\mathrm{pH} 3,5$ et 9 et il existe une relation linéaire entre le logarithme de la vitesse de gélification et le $\mathrm{pH}(6$ à 9$)$. La contrainte de compression des gels n'évolue pas linéairement avec le taux de compression (0 à 22\%). L'élasticité, estimée à partir du module d'Young apparent $\left(\mathrm{E}_{\mathrm{a}}\right)$ calculé à un faible taux de compression $(2 \%)$, augmente avec la concentration en protéines, la température et le temps de chauffage. $A 10 \%$ de protéines, $E_{a}$ est le plus élevé à pH $4(191 \mathrm{kPa})$ et $10(154 \mathrm{kPa})$, et le plus faible à $\mathrm{pH} 5(12 \mathrm{kPa})$ et $11(24 \mathrm{kPa})$. La fermeté, calculée à partir de la contrainte à $22 \%$ de compression, est la plus élevée à pH 10 $(>18 \mathrm{kPa})$ et $8(16 \mathrm{kPa})$, et la plus faible à pH $5(3 \mathrm{kPa})$ et $4(7 \mathrm{kPa}) . \mathrm{A} \mathrm{pH} 4$, le gel est translucide et très fragile. Entre $\mathrm{pH} 5$ et 9 , il est blanc et opaque. $\mathrm{A} \mathrm{pH} 11$, il est le plus translucide.
\end{abstract}

concentré de protéines de lactosérum / gélification thermique / viscosité / fermeté / module d'Young

Summary - Rheological characterization and heat gelation of whey protein concentrate. Rheological behaviour and gelation kinetic of defated and diafiltered whey protein concentrate (WPC) have been studied with a coaxial cylinder viscometer. Rheological characteristics of gels have been measured with a compression rheometer. The solutions of the WPC containing 4-14\% at $25^{\circ} \mathrm{C}$ and at $\mathrm{pH} 7$ has a newtonian behaviour for the shear rate between 82 and $955 \mathrm{~s}^{-1}$. The logarithm of the viscosity is a linear function of the protein concentration. The activation energy of flow increases linearly with protein concentration from about $13 \mathrm{~kJ} \cdot \mathrm{mol}^{-1}$ for water to $20 \mathrm{~kJ}^{\circ} \mathrm{mol}^{-1}$ for $14 \%$ protein solution. At $10 \%$ protein and $60^{\circ} \mathrm{C}$, the heat gelling solutions have shear thinning behaviour. At $60^{\circ} \mathrm{C}$, the increase of the apparent viscosity is the fastest at $\mathrm{pH} 4$ and 9 . At $70^{\circ} \mathrm{C}$, gelation is observed from $\mathrm{pH} 3.5-9$ and the logarithm of the gelation velocity is a linear function of $\mathrm{pH}$ between 6 and 9. The relation between the compression ratio (0-22\%) of the protein gels and the compression stress is non-linear. The elasticity, estimated as the apparent Young's modulus $\left(E_{a}\right)$ calculated for low compression ratio (2\%), increases with protein concentration, temperature and time of heating. For the gels containing $10 \%$ protein, the highest level of $E_{a}$ is observed for the $\mathrm{pH} 4(191 \mathrm{kPa})$ and $10(154 \mathrm{kPa})$, the lowest for the $\mathrm{pH} 5(12 \mathrm{kPa})$ and 11 (24 kPa). The firmness, estimated by the 
stress at the compression ratio of $22 \%$, is the highest at $\mathrm{pH} 10(>18 \mathrm{kPa})$ and $8(16 \mathrm{kPa})$, and the lowest at $\mathrm{pH} 5(3 \mathrm{kPa})$ and $\mathrm{pH} 4(7 \mathrm{kPa})$. At $\mathrm{pH} 4$, the gel is translucent and very brittle. For the $\mathrm{pH}$ between 5 and 9 , it is white and opaque. At 11, the gel is the most trans/ucent.

\section{whey protein concentrate / heat gelation / viscosity / firmness / Young's modulus}

\section{INTRODUCTION}

L'aptitude à la gélification thermique est, parmi les propriétés fonctionnelles des concentrés de protéines de lactosérum (CPL), une des plus recherchées en industrie alimentaire (Kinsella, 1984). La gélification du CPL consiste en la mise en place d'un réseau protéique tridimensionnel ordonné et suffisamment ouvert pour emprisonner beaucoup d'eau, ce qui nécessite un équilibre critique entre les forces répulsives et attractives intermoléculaires dépendant du $\mathrm{pH}$ (Mulvihill et Kinsella, 1987). A pH acide et donc au $\mathrm{pHi}$ des séroprotéines (voisin de 5), les liaisons de type hydrophobe, hydrogène et ionique sont principalement responsables de ce phénomène; au-delà de $\mathrm{pH} 6,8$, les ponts disulfures, résultant de la dissociation et du réarrangement des groupements SH/SS, interviendraient de façon prépondérante (De Wit et De Boer, 1975; De Wit, 1981).

De nombreux critères ont été proposés pour évaluer l'aptitude à la gélification thermique des CPL. Hagett (1976), Johns et Ennis (1981), Hillier et Cheeseman (1979) et Sternberg et al (1976) évaluent, par l'écoulement gravitationnel, le temps, la température et la concentration en protéines nécessaires pour obtenir un gel : il y a gélification lorsque l'inversion du tube contenant la solution protéique ne provoque pas d'écoulement. Des méthodes instrumentales sont également utilisées telles que les mesures de viscosité en régime permanent (McDonough et al, 1974), les mesures du module complexe en ré- gime dynamique (Paulsson et al, 1986), la pénétrométrie (Hagett, 1976), la compression (Johns et Ennis, 1981; Sternberg et al, 1976; Mulvihill et Kinsella, 1988; Zirbel et Kinsella, 1988). Les propriétés physiques du gel sont aussi déterminées par analyse sensorielle (Cooper et al, 1977) avec comparaison à des gels standard (Dunkerley et Hayes, 1980), et enfin par microscopie électronique à balayage (Mulvihill et Kinsella, 1988; Harwalkar et Kalab, 1985). Les résultats varient selon les auteurs en raison de la diversité des méthodes mises en œuvre et de la composition du CPL (concentration en protéines, en ions, en lipides et en lactose), de l'état des protéines et du $\mathrm{pH}$ de la solution qui dépendent du procédé de préparation du CPL (Morr, 1979; Kinsella, 1984; Mulvihill et Kinsella, 1987).

La gélification thermique des séroprotéines nécessite une concentration minimale en protéines qui dépend $\mathrm{du} \mathrm{pH}$. Hillier et Cheeseman (1979) ont trouvé une concentration minimale de $8 \%$ à pH 8 à partir d'un CPL obtenu par chromatographie sur échangeur d'ions. Selon Sternberg et al (1976), qui ont travaillé sur un CPL obtenu par précipitation avec de l'acide polyacrylique, elle est de $1 \%, 1,4 \%$, $4,4 \%$ et $4,8 \%$ de protéines, respectivement, à pH 4,5,6,0,8,0 et 5,0. L'augmentation de la concentration en protéines audelà de cette valeur critique, de la température de chauffage $\left(60\right.$ à $\left.90^{\circ} \mathrm{C}\right)$ et du $\mathrm{pH}$ (6 à 9) diminuent le temps nécessaire à la gélification (Hillier et Cheeseman, 1979; Hillier et al, 1980), alors que la présence de lipides retarde ce phénomène (Stern- 
berg et al, 1976). Cooper et al (1977) ont montré à partir de l'évaluation sensorielle que la fermeté du gel, le cassant, le brillant et la couleur dépendent du $\mathrm{pH}$, de la concentration en protéines, en $\mathrm{CaCl}_{2}$, en $\mathrm{NaCl}$ et en saccharose. L'élévation du $\mathrm{pH}$ entre 6 et 8 , et de la température de chauffage entre 70 et $90^{\circ} \mathrm{C}$, augmente la fermeté du gel de CPL obtenu par chromatographie sur échangeur d'ions (Zirbel et Kinsella, 1988). Selon Dunkerley et Zadow (1981), les gels de concentré de protéines de lactosérum doux présentent une fermeté moyenne à $\mathrm{pH} 3$ et 6 , excellente à $\mathrm{pH} 8$ et la déminéralisation entraîne une diminution de l'aptitude à la gélification à $\mathrm{pH} 3$.

La mise au point récente par Fauquant et al (1985a, b) d'un procédé de délipidation de ces protéines par microfiltration et de concentration par ultrafiltration et diafiltration (jusqu'à $90 \%$ de pureté) offre de nouvelles possibilités de valorisation du lactosérum. L'objet de ces travaux consiste à étudier le pouvoir gélifiant d'un tel concentré protéique afin d'envisager son incorporation dans différents aliments, en substitution par exemple au blanc d'œuf. Après avoir caractérisé ce produit sur le plan rhéologique, nous nous sommes efforcés d'étudier l'influence du $\mathrm{pH}$, de la concentration en protéines et du traitement thermique sur la cinétique de gélification thermique et la texture des gels obtenus.

\section{MATÉRIELS ET MÉTHODES}

\section{Produits}

La poudre de concentré de protéines de lactosérum présure (CPL), délipidé selon le procédé mis au point par Fauquant et al (1985b) était fournie par l'entreprise EURIAL (Herbignac, France). La composition chimique du CPL est présentée dans le tableau I. Quelques mesures comparatives ont été réalisées avec du blanc d'œuf congelé (12,2\% d'extrait sec total, $10,5 \%$ de la matière azotée totale, $\mathrm{pH} 8,25)$, fourni par l'entreprise A B C D (Ploërmel, France).

\section{Reconstitution du CPL}

La poudre de CPL était ajoutée en quantités variables dans de l'eau désionisée à $30^{\circ} \mathrm{C}$. La solubilisation était réalisée en $90 \mathrm{~min}$ avec une agi-

Tableau I. Composition chimique du concentré de protéines de lactosérum en poudre (CPL). Chemical composition of the whey protein concentrate powder (WPC).

\begin{tabular}{llc} 
Composants & Components & Composition $(g / 100 \mathrm{~g})$ \\
\hline \multirow{2}{*}{ Extrait sec total (EST) } & Dry matter (DM) & \\
Matière azotée totale (MAT) & Total nitrogen (TN) & 95,3 \\
Azote non protéique (NPN) & Non protein nitrogen (NPN) & 84,8 \\
MAT/EST & TN/DM & 4,65 \\
Matière grasse (MG) & Fat & 88,9 \\
Lactose & Lactose & 2,7 \\
Cendres & Ashes & 3,8 \\
Ca & Ca & 2,5 \\
K & $K$ & 0,72 \\
Na & Na & 0,35 \\
$\beta$-Lactoglobuline & $\beta$-Lactoglobulin & 0,18 \\
$\alpha$-Lactalbumine & $\alpha$-Lactalbumin & 60,4 \\
& & 13,4 \\
\hline
\end{tabular}


tation modérée. Pour une concentration en protéines de $10 \%$ (poids/poids), le pH de la solution était de 6,5. Les ajustements de $\mathrm{pH}$ étaient réalisés à l'aide d'acide chlorhydrique $\mathrm{HCl}$ et de soude $\mathrm{NaOH}$ de normalités égales à 10 .

\section{Analyses}

La teneur en extrait sec total (EST) de la poudre était déterminée par dessication à l'étuve à $102-105^{\circ} \mathrm{C}$ pendant $5 \mathrm{~h}$, selon la norme DSV donnée par Serres et al (1973). L'analyse quantitative des lipides était effectuée selon la méthode SBR (FIL, 1959), celle du lactose par la méthode proposée par Acton (1977). La détermination des teneurs en matières azotées totales (MAT) était réalisée par la méthode macro-Kjeldahl. Le dosage de l'azote non protéique (NPN) était effectué par dosage de l'azote total dans le surnageant après précipitation de la totalité des protéines en présence d'acide trichloroacétique à $12 \%$ selon la méthode de Rowland (1938). Les teneurs en cendres étaient déterminées par incinération de la matière sèche dans un four à $530^{\circ} \mathrm{C}$, selon la norme DSV donnée par Serres et al (1973). La détermination des teneurs en éléments minéraux était réalisée par absorption atomique (Brulé et al, 1974). Le dosage de la $\beta$ lactoglobuline et de l' $\alpha$-lactalbumine était effectué par chromatographie liquide haute pression (Varian, USA : colonne TSK 3000 SW, tampon tris $0,01 \mathrm{~mol} / \mathrm{l}, \mathrm{pH} 6,7$, débit $1 \mathrm{ml} / \mathrm{min}$, détection $280 \mathrm{~nm}$ ). Les déterminations de $\mathrm{pH}$ étaient réalisées avec un appareil type PHN-850 (Tacussel, France). La solubilité protéique a été estimée selon la méthode décrite par Morr et al (1985), par détermination de la MAT du surnageant après centrifugation (20000 g, $30 \mathrm{~min}$ ) d'une solution contenant $1 \%$ de protéines diluées dans du $\mathrm{NaCl}$ à $0,1 \mathrm{~mol} / /$.

\section{Viscosité}

Les mesures de viscosité étaient réalisées à l'aide d'un viscosimètre rotatif à cylindres coaxiaux type Rhéomat 30 (Contraves, Suisse). Les diamètres des cylindres extérieur et intérieur étaient de 50 et $49 \mathrm{~mm}$ respectivement. Le viscosimètre était relié à un microordinateur type Apple II+ (Apple Computer Inc, Etats-Unis) par l'intermédiaire d'une interface convertisseur analogique numérique type CAN-1612M1 (MID, France). Un programme d'acquisition de données (Korolczuk et al, 1985) a été utilisé. Le temps d'intégration du signal était de 0,5 s.

L'échantillon était placé dans la cellule de mesure à température ambiante puis plongé dans le bain d'eau porté à la température désirée $\left( \pm 0,1^{\circ} \mathrm{C}\right)$. Les mesures étaient réalisées, après un temps d'attente de $2 \mathrm{~min}$, à différentes températures $\left(10\right.$ à $70{ }^{\circ} \mathrm{C}$ ) et vitesses de cisaillement ( 82 à $\left.955 \mathrm{~s}^{-1}\right)$.

\section{Gélification}

La cinétique de gélification a été étudiée à partir des mesures de viscosité, en fonction de la durée de chauffage à $60^{\circ} \mathrm{C}$ et à $70^{\circ} \mathrm{C}$. La saisie des mesures commençait dès l'immersion du corps de mesure dans le bain d'eau.

Le test de compression a été effectué à l'aide du rhéomètre digital, mis au point par CardenasCaroti et al (1985), sur des gels préparés de la façon suivante :

- cristallisoir de $120 \mathrm{ml}$ (hauteur : $38 \mathrm{~mm}$; diamètre: $66 \mathrm{~mm}$ ), contenant $100 \mathrm{~g}$ de solution protéique, immergé dans un cristallisoir de 250 $\mathrm{ml}$ contenant $180 \mathrm{~g}$ d'eau, à une température de $25^{\circ} \mathrm{C}$;

- chauffage (cristallisoir sans couvercle) par micro-ondes jusqu'à la température souhaitée $\left(80^{\circ} \mathrm{C}\right.$ et $90^{\circ} \mathrm{C}$ soit respectivement 110 s et 130 $s$ de chauffage);

- chauffage (cristallisoir + couvercle) dans un bain d'eau pendant un temps $t$ (10 et 30 $\mathrm{min})$, température du bain d'eau de $80^{\circ} \mathrm{C}$ ou $90^{\circ} \mathrm{C}$;

- refroidissement 10 min dans l'eau glacée;

- à température ambiante pendant une nuit (environ 16 heures);

- test de compression à température ambiante (environ $20^{\circ} \mathrm{C}$ ) sur les gels non démoulés (hauteur : $27,5 \mathrm{~mm}$; diamètre : $66 \mathrm{~mm}$ ).

La puissance absorbée par le four à microondes (Micromat 700, AEG) était de 1400 watts, celle restituée de 650 watts, et sa capacité volumique de 32 litres. 
Le principe du test de compression consiste à suivre l'évolution dans le temps de la force de compression du gel à vitesse constante $(4 \mathrm{~mm} /$ min). Le gel est comprimé entre un plateau ( $50 \mathrm{~mm}$ de diamètre) suspendu à un capteur de $125 \mathrm{~N}$, et le socle du rhéomètre. La contrainte de compression (S) et le module d'Young apparent $\left(E_{a}\right)$ ont été calculés à partir de ce test :

et

$$
S=F / A
$$

$$
E_{a}=S \cdot h_{a} / \Delta h
$$

ou : $S=$ contrainte en $\mathrm{Pa}$,

$\mathrm{F}=$ force en $\mathrm{N}$,

$A=$ surface du plateau en $\mathrm{m}^{2}$,

$E_{a}=$ module d'Young apparent en $\mathrm{Pa}$,

$h_{o}=$ hauteur initiale du gel en $\mathrm{m}$,

$\Delta h=$ variation de hauteur en $\mathrm{m}$,

$\Delta h / h_{o}=$ déformation relative ou taux de compression.

La reproductibilité des mesures a été déterminée sur 4 séries de 4 gels obtenus à partir d'une solution à $10 \%$ de protéines et à $\mathrm{pH} 7$, ayant subi une montée en température de 25 à $90{ }^{\circ} \mathrm{C}$ au four à micro-ondes. Le coefficient de variation du module d'Young apparent et de la contrainte maximale de compression $\left(S_{m}\right)$ est respectivement de $9 \%$ ef $10 \%$.

\section{RÉSULTATS ET DISCUSSION}

\section{Solubilité}

A $20^{\circ} \mathrm{C}$, la plus faible valeur de solubilité a été observée à pH 5 (94\% d'azote soluble par rapport à l'azote total), $\mathrm{pH}$ voisin du $\mathrm{pHi}$ où les répulsions interprotéiques sont minimales. Aux pH situés de part et d'autre de 5 , celle-ci augmente à $94,4,95,3$ et $96 \%$ à respectivement $\mathrm{pH} 3,7$ et 9 . Ces valeurs sont supérieures à celles déterminées par Morr et al (1985) sur un CPL obtenu à partir de l'ultrafiltration d'un lactosérum doux ( 85 et $91,4 \%$ de protéines solubles à respectivement $\mathrm{pH} 3$ et 7 ). A
$10 \%$ de protéines (MAT-NPN), la diminution de la solubilité à pH 5 est perceptible visuellement puisqu'elle se traduit par un aspect plus trouble de la solution. L'augmentation de la solubilité avec l'éloignement du $\mathrm{pH}$ au $\mathrm{pHi}$, liée à l'augmentation des forces répulsives interprotéiques (Kinsella, 1984), améliore le caractère translucide de la solution.

\section{Viscosité}

A une température de $25^{\circ} \mathrm{C}$, pour une concentration protéique (MAT-NPN) comprise entre 4 et $14 \%$, à $\mathrm{pH} 7$ et pour des vitesses de cisaillement comprises entre 82 et $955 \mathrm{~s}^{-1}$, le comportement du produit est newtonien car la viscosité est indépendante de la vitesse de cisaillement. Le logarithme de la viscosité des solutions est proportionnel à la concentration en protéines C (en \%) :

$$
\begin{gathered}
\log \eta=-0,128+0,053 \cdot C ; \\
r=0,996 ; n=25
\end{gathered}
$$

L'élévation de la teneur en protéines entraîne une augmentation de la viscosité du CPL que l'on peut attribuer à l'effet "concentration", c'est-à-dire au rapprochement des éléments protéiques qui favorise les interactions entre ceux-ci (Lee et Rha, 1979). A $10 \%$ de protéines, la viscosité est indépendante du $\mathrm{pH}$ entre 3 et 10 , et le comportement rhéologique du produit est également newtonien.

A pH 7, la viscosité diminue avec l'augmentation de la température (fig 1). Entre $10^{\circ} \mathrm{C}$ et $60^{\circ} \mathrm{C}$, la viscosité passe de 6,7 à $2,3 \mathrm{mPa} \cdot \mathrm{s}$ pour une concentration en protéines de $14 \%$, et de 1,74 à $0,7 \mathrm{mPa} \cdot \mathrm{s}$ pour une concentration de $4 \%(\dot{\gamma}=517$ $\mathrm{s}^{-1}$ ). Entre $10^{\circ} \mathrm{C}$ et $50^{\circ} \mathrm{C}$, le logarithme de la viscosité augmente de façon linéaire en fonction de l'inverse de la température ab- 


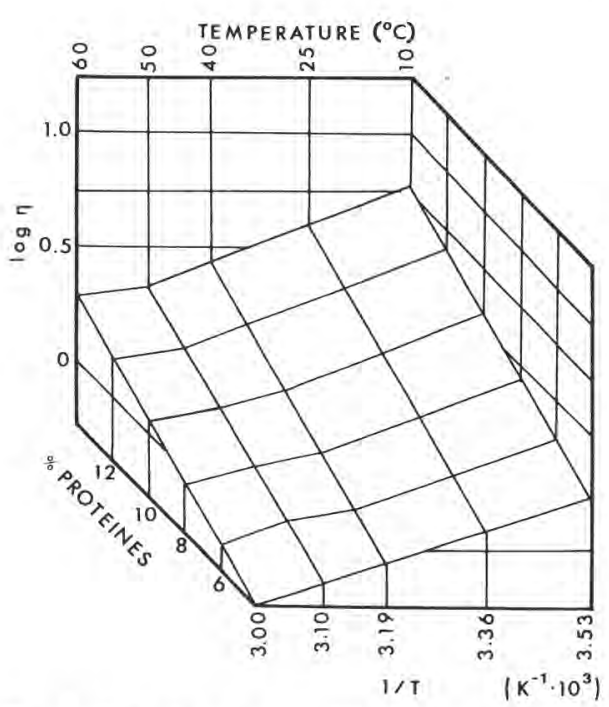

Fig 1. Évolution du logarithme de la viscosité ( $\eta$ ) du CPL en fonction de la concentration en protéines (\%) et de l'inverse de la température absolue (T), à pH 7 et $\dot{\gamma}=517 \mathrm{~s}^{-1}$.

Evolution of the logarithm of the viscosity (7) of the whey protein concentrate as a function of the protein concentration (\%) and of the reciprocal absolute temperature (T) at $\mathrm{pH} 7$ and $\dot{\gamma}=$ $517 \mathrm{~s}^{-1}$.

solue $(1 / T):(r=0,997 \pm 0,12 \%)$ quelle que soit la concentration en protéines. Entre 50 et $60^{\circ} \mathrm{C}$, l'évolution de la viscosité ne suit plus l'équation d'Andrade (1930). L'insolubilisation partielle des protéines, liée à l'augmentation des interactions hydrophobes interprotéiques sous l'effet de la température (De Wit et Klarenbeek, 1984) pourrait expliquer ce résultat.

A partir de l'équation d'Andrade, nous avons calculé entre 10 et $50^{\circ} \mathrm{C}$ l'énergie apparente d'activation d'écoulement de l'eau et de la solution de CPL à différentes concentrations en protéines :

$$
\log \eta=\mathrm{B}+\frac{E_{e}}{\mathrm{R} \cdot T}
$$

ou $\eta=$ viscosité (mPa*s),

$B=$ constante,

$E_{\theta}=$ énergie apparente d'activation d'écoulement $\left(\mathrm{J} \cdot \mathrm{mol}^{-1}\right)$,

$\boldsymbol{R}=$ constante des gaz parfaits $(8,3143$ $\mathrm{J} \cdot \mathrm{mol}^{-1} \cdot \mathrm{K}^{-1}$, ,

$T=$ température absolue (en K).

Pour les concentrations en protéines de $4,6,8,10,12$ et $14 \%, E_{e}$ est égale respectivement à $16,4,16,1,16,9,18,5,19,8$ et $20,3 \mathrm{~kJ} \cdot \mathrm{mol}^{-1}$. Ces valeurs sont supérieures à celle de l'eau calculée à partir des valeurs de viscosité $\left(16,0 \mathrm{~kJ} \cdot \mathrm{mol}^{-1}\right)$ mesurées par Bingham et Jackson (1918), ou à partir des mesures de viscosité réalisées avec le viscosimètre Rhéomat 30 $\left(13,6 \mathrm{~kJ} \cdot \mathrm{mol}^{-1}\right)$. La différence entre ces 2 valeurs de $E_{e}$ de l'eau provient du manque de sensibilité du Rhéomat 30 , et d'ailleurs d'une façon générale des viscosimètres de rotation classiques pour les faibles viscosités, comme le soulignent Wayne et Shoemaker (1988). Pour les teneurs en protéines supérieures ou égales à $6 \%$, la relation entre l'énergie d'activation d'écoulement $\left(E_{e}\right)$ et la concentration de protéines (C, en \%) est linéaire :

$E_{e}=12,670+0,565 \cdot C ; r=0,988 ; n=5(5)$

\section{Gélification}

La figure 2 montre l'évolution de la viscosité en fonction du temps au cours de la gélification thermique à $60^{\circ} \mathrm{C}$ d'une solution à $\mathrm{pH} 4$ et à $10 \%$ de protéines. Cette courbe se décompose en 3 phases :

phase 1 : la viscosité augmente de façon exponentielle;

phase 2 : la viscosité augmente de façon linéaire;

phase 3 : la viscosité plafonne ou même chute. 


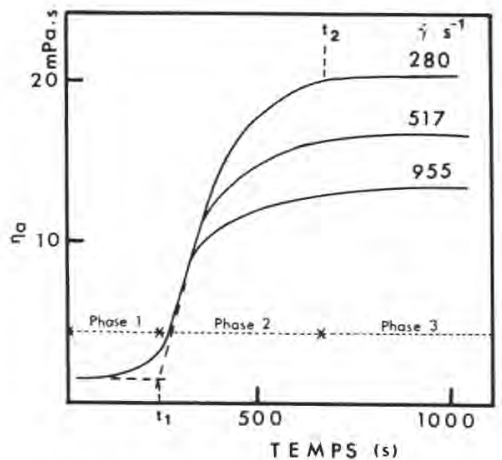

Fig 2. Evolution de la viscosité apparente du CPL à $10 \%$ de protéines au cours de la gélification thermique à $60^{\circ} \mathrm{C}$ et à $\mathrm{pH} 4$ en fonction de la vitesse de cisaillement $(\dot{\gamma})$ de 280 à $955 \mathrm{~s}^{-1}$.

Evolution of the apparent viscosity of the whey protein concentrate at $10 \%$ of protein during heat induced gelation at $60^{\circ} \mathrm{C}$ and $\mathrm{pH} 4$ as a function of the shear rate $(\dot{\gamma})$ from $280-955 \mathrm{~s}^{-1}$.

Contrairement à la viscosité des solutions avant gélification, celle des solutions en cours de gélification est dépendante de la vitesse de cisaillement : le produit présente un comportement rhéofluidifiant, c'est-à-dire que la viscosité diminue avec l'augmentation de la vitesse de cisaillement. La gélification a été étudiée à une vitesse de cisaillement de $280 \mathrm{~s}^{-1}$ permettant d'une part de se situer au-dessus de la limite inférieure de sensibilité du viscosimètre lors du début de la phase 1 , et d'autre part d'avoir la plus grande amplitude de variation de la viscosité apparente dans les conditions de mesure établies.

La concentration minimale pour laquelle on observe une augmentation de la viscosité apparente lors d'un chauffage à $70^{\circ} \mathrm{C}$ se situe entre $3-4 \%$ à $\mathrm{pH} 4$, et entre $6-7 \%$ à pH 9 (fig 3). Sternberg et al (1976) ont obtenu des résultats similaires mais toutefois avec des concentrations inférieures. Le mode d'obtention du CPL (précipitation avec de l'acide polyacrylique), la méthode

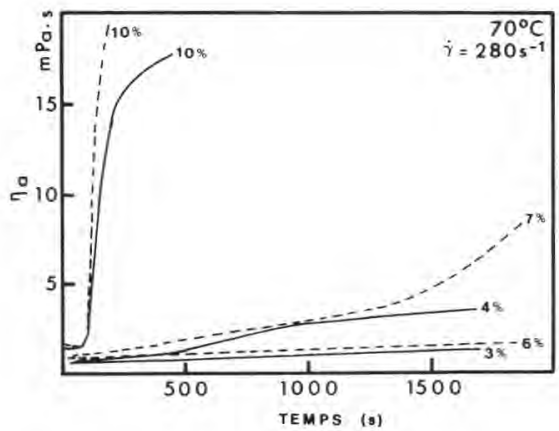

Fig 3. Détermination de la concentration protéique minimale de gélification à $70^{\circ} \mathrm{C}$ à partir des mesures de viscosité apparente à $\mathrm{pH} 4$ (lignes continues) et à pH 9 (lignes pointillées). La teneur en protéines (en \%) des solutions de CPL est indiquée sur la figure. Vitesse de cisaillement $\dot{\gamma}=280 \mathrm{~s}^{-1}$.

Determination of the minimal protein concentration of gelation at $70{ }^{\circ} \mathrm{C}$ by measurement of the apparent viscosity at $\mathrm{pH} 4$ (continuous line) and at $\mathrm{pH} 9$ (broken line). Protein content (\%) of the whey protein concentrate is indicated in the figure. The shear rate $\dot{\gamma}=280 \mathrm{~s}^{-1}$.

quasi non destructive d'appréciation de la gélification basée sur l'écoulement gravitationnel de la solution protéique après chauffage, de même que le traitement thermique plus intense $\left(100^{\circ} \mathrm{C}\right.$ pendant 30 min) mis en œuvre par ces auteurs sont probablement à l'origine de cette différence de résultats.

Pour une teneur en protéines de $10 \%$, le $\mathrm{CPL}$ gélifie dès $60^{\circ} \mathrm{C}$ à $\mathrm{pH} 3,5,4,4,5,8$ et 9 (fig 4) et à partir de $70^{\circ} \mathrm{C}$ de $\mathrm{pH} 5$ à 7,5 (figs 5 et 6 ). Les températures de gélification définies par Burgess et Kelly (1979) sur des concentrés à $11 \%$ de protéines obtenus par échanges d'ions (CPI) et par ultrafiltration de lactosérum non délipidé (CPLND) sont respectivement de $58^{\circ} \mathrm{C}$ à $\mathrm{pH} 7$ et $56^{\circ} \mathrm{C}$ à pH9, et de $74^{\circ} \mathrm{C}$ à pH7 et $67^{\circ} \mathrm{C}$ à pH9. Selon ces auteurs, les lipides sont responsables de ces différences : li- 


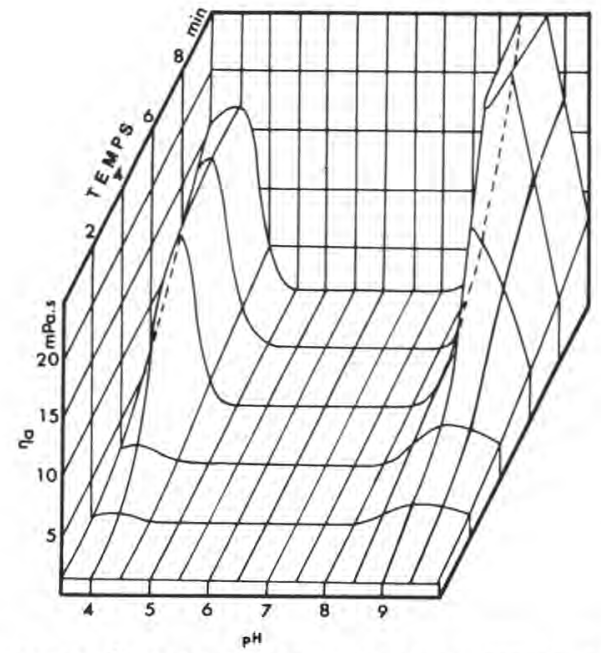

Fig 4. Évolution de la viscosité apparente du CPL à $10 \%$ de protéines au cours de la gélification thermique à $60^{\circ} \mathrm{C}$ en fonction du temps et du $\mathrm{pH}$. Vitesse de cisaillement $\dot{\gamma}=280 \mathrm{~s}^{-1}$. Evolution of the apparent viscosity of the whey protein concentrate at $10 \%$ protein during heat induced gelation at $60^{\circ} \mathrm{C}$ as' a function of time and $\mathrm{pH}$. The shear rate $\dot{\gamma}=280 \mathrm{~s}^{-1}$.

pides/protéines solubles $=0,53 \%$ du CPI et $8,6 \%$ du CPLND. Les résultats obtenus dans notre étude confortent d'ailleurs cette hypothèse puisque pour un rapport L/PS de $3,3 \%$ pour le CPL étudié, les températures minimales de gélification sont intermédiaires à celles obtenues par Burgess et Kelly (1979).

Pour une solution à $10 \%$ de protéines et à $60^{\circ} \mathrm{C}$ (fig 4), c'est à $\mathrm{pH} 4$ et 9 que la solution gélifie le plus rapidement. A $70^{\circ} \mathrm{C}$, l'élévation de la viscosité apparente de la solution de protéines en fonction du temps de chauffage est plus rapide qu'à $60^{\circ} \mathrm{C}$ quel que soit le $\mathrm{pH} . \mathrm{A} \mathrm{pH}<5$ (fig 5), c'est à $\mathrm{pH} 4$ et 4,5 que la vitesse de gélification est la plus élevée, mais les gels sont très fragiles et se destructurent rapidement sous l'effet du cisaillement. La prédominance, dans la structure des gels ob-

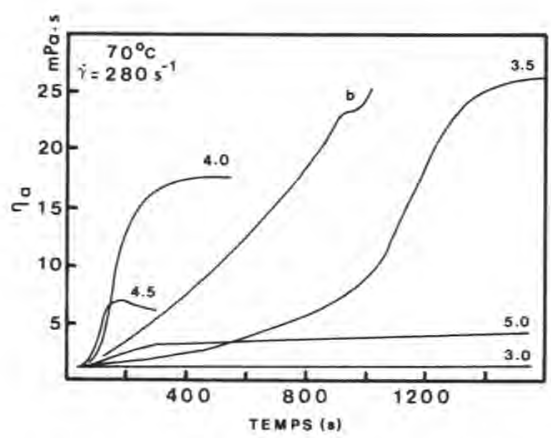

Fig 5. Évolution de la viscosité apparente (na) du CPL à $10 \%$ de protéines au cours de la gélification à $70^{\circ} \mathrm{C}$ en fonction du temps et du $\mathrm{pH}$. Les valeurs au-dessus des courbes représentent le $\mathrm{pH}$ des solutions protéiques. Vitesse de cisaillement $\dot{\gamma}=280 \mathrm{~s}^{-1}, \mathrm{~b}=$ blanc d'cuf à $\mathrm{pH}$ 8,25 .

Evolution of the apparent viscosity (ma) of the whey protein concentrate at $10 \%$ protein during heat induced gelation at $70^{\circ} \mathrm{C}$ as a function of time and $\mathrm{pH}$. The values over the curves represent the $\mathrm{pH}$ of protein solutions. The shear rate $\dot{\gamma}=280 \mathrm{~s}^{-1}, b=e g g$ white at $\mathrm{pH} 8.25$.

tenus à $\mathrm{pH}$ acide, de liaisons non covalentes de faible énergie (hydrogène, hydrophobe, ionique; De Wit et De Boer, 1975) et donc plus sensibles au cisaillement inhérent aux mesures de viscosité, explique ce résultat. $\mathrm{A} \mathrm{pH}>5$ (fig 6), la viscosité augmente d'autant plus rapidement que le $\mathrm{pH}$ est proche de 9. Les groupements sulfhydriles libres (SH), notamment ceux de la $\beta$-lactoglobuline qui possède un groupement SH libre par molécule, sont plus réactifs au-dessus de pH 6,8 (De Wit, 1981), ce qui favorise leur réarrangement en ponts S-S intermoléculaires (De Wit et De Boer, 1975; De Wit, 1981). Ces liaisons covalentes de forte énergie renforcent la structure du gel, et, selon Zirbel et Kinsella (1988), elles sont d'autant plus nombreuses que le $\mathrm{pH}$ est alcalin entre $\mathrm{pH} 6$ et 8. De plus, l'augmentation de l'hydratation des protéines liée à l'augmentation du 
degré d'ionisation des groupements $\mathrm{COOH}$ des protéines avec l'élévation de $\mathrm{pH}$ a probablement une influence sur l'évolution de la viscosité du CPL. Hillier et al (1980) ont montré à partir d'une méthode visuelle que le temps nécessaire à l'obtention du gel diminue avec l'abaissement du $\mathrm{pH}$ entre $\mathrm{pH}$ 9 et 6 . Ces travaux sont complémentaires des nôtres et nous permettent de supposer que ce sont des liaisons non covalentes qui se mettent tout d'abord en place dans le réseau protéique, d'autant plus aisément que le $\mathrm{pH}$ est proche de 5 . Le cisaillement relatif aux mesures de viscosité détruit ce type de liaison de faible énergie. L'augmentation de la viscosité reflète alors principalement la formation de ponts S-S intermoléculaires dans cette gamme de $\mathrm{pH}$. Ces résultats posent donc clairement le problème de l'étude de la gélification thermique des CPL à partir de la viscosimétrie

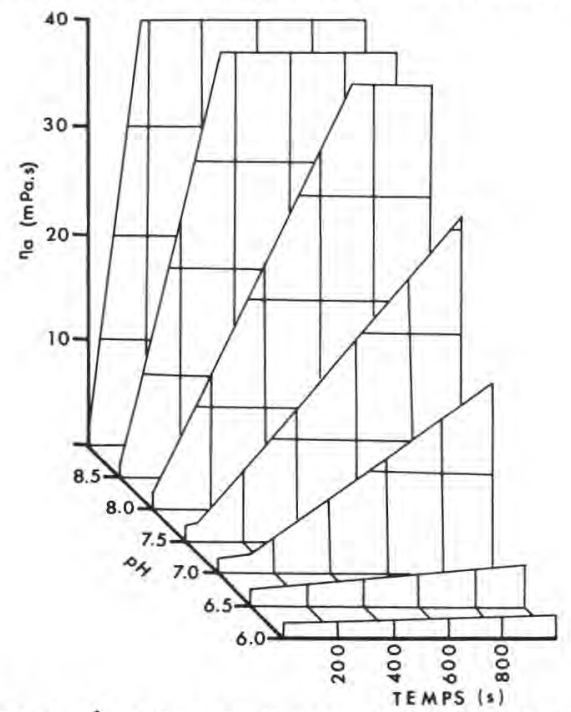

Fig 6. Evolution de la viscosité apparente du CPL à $10 \%$ de protéines au cours de la gélification à $70^{\circ} \mathrm{C}$ en fonction du temps et du pH. Vitesse de cisaillement $\dot{\gamma}=280 \mathrm{~s}^{-1}$.

Evolution of the apparent viscosity of the whey protein concentrate at $10 \%$ protein during heat induced gelation at $70^{\circ} \mathrm{C}$ as a function of time and $\mathrm{pH}$. Shear rate $\dot{\gamma}=280 \mathrm{~s}^{-1}$. de rotation. II serait souhaitable d'étudier ce phénomène à partir de mesures réalisées en régime dynamique (Paulson et al, 1986), qui n'affectent pas, semble-t-il, le processus de gélification.

Pour les $\mathrm{pH}$ compris entre 6 et 9 , à $70^{\circ} \mathrm{C}$ (fig 6), l'augmentation de la viscosité apparente est une fonction linéaire du temps de chauffage. II existe également une relation linéaire entre le logarithme de l'indice de la vitesse de gélification ( $\log k$ ), représenté par la pente $k$ des droites de la figures 6 , et le $\mathrm{pH}$ des solutions de protéines (fig 7):

$$
\begin{aligned}
\log k & =-4,711+0,813 \cdot \mathrm{pH} ; \\
r & =0,990 ; n=7
\end{aligned}
$$

où $k$ est exprimé en $\mu \mathrm{Pa}$.

A $70^{\circ} \mathrm{C}$, la cinétique de gélification du blanc d'œuf à $\mathrm{pH} 8,25$ est intermédiaire entre celles observées pour les CPL à pH 3,5 et 4 (fig 5), et à pH 7 et 7,5 (fig 6).

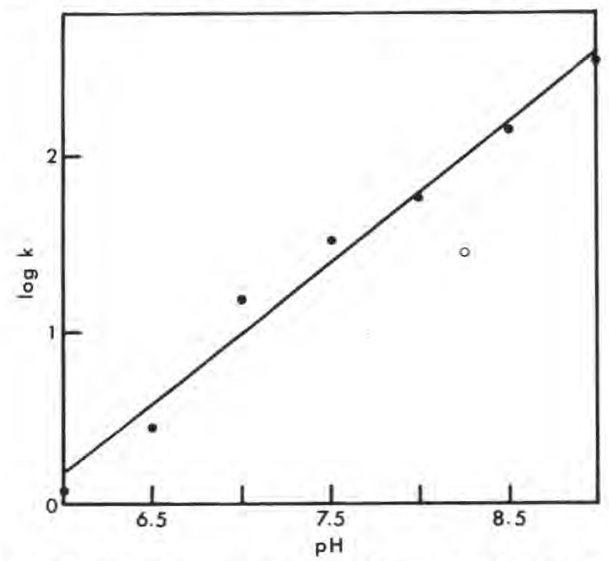

Fig 7. Relation entre le logarithme de l'indice de la vitesse d'augmentation de la viscosité apparente ( $k$ en $\mu \mathrm{Pa}$ ) et le $\mathrm{pH}$ (équation (6)) au cours de la gélification à $70^{\circ} \mathrm{C}$ de solutions à $10 \%$ de protéines. $0=$ blanc d'œuf à pH 8,25.

The logarithm of the velocity index of the apparent viscosity increase ( $\mathrm{k}$ in $\mu \mathrm{Pa}$ ) as a function of $\mathrm{pH}$ (equation (6)) during heat induced gelation at $70^{\circ} \mathrm{C}$ at $10 \%$ protein solutions. $0=e g g$ white at $\mathrm{pH} 8.25$. 


\section{Caractérisation rhéologique des gels}

Comme le montre la figure 8 , la contrainte des gels obtenus à partir d'une solution à $10 \%$ de protéines, ayant subi une montée en température de 25 à $90^{\circ} \mathrm{C}$ dans le four à micro-ondes, augmente avec le taux de compression. Pour un gel obtenu à $\mathrm{pH} 4$, elle présente un maximum vers un taux de compression de $10 \%$, puis diminue jusqu'à un taux de compression de $22 \%$. Entre $\mathrm{pH}$ 5 et 9 , nous observons une augmentation quasi linéaire de la contrainte.

$\mathrm{A} \mathrm{pH} 7$ et à $90^{\circ} \mathrm{C}$, le module d'Young apparent à $2 \%$ de compression $\left(E_{a}\right)$ et la contrainte à $22 \%$ de compression $\left(S_{m}\right)$ du gel augmentent avec l'élévation de la concentration en protéines (fig 9). Avec entre 4 et $6 \%$ de protéines, $E_{a}$ et $S_{m}$ augmentent respectivement de 200 et $50 \%$. Cette augmentation devient maximale

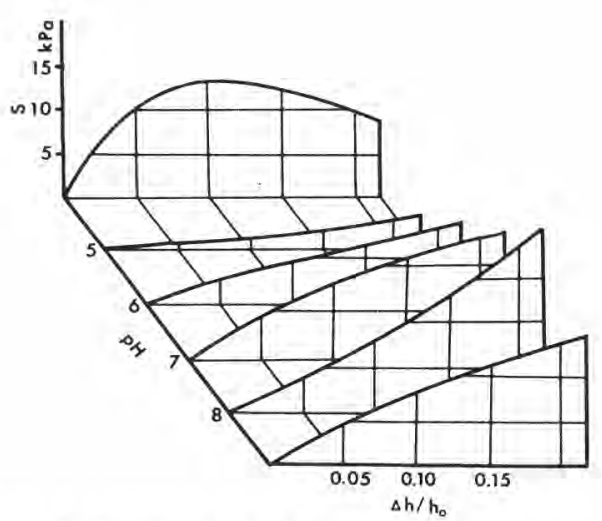

Fig 8. Évolution de la contrainte de compression en fonction du taux de compression $(\Delta h$ ) $h_{o}$ ) et du pH des solutions du CPL. Les gels ont été obtenus à partir d'un concentré à $10 \%$ de protéines ayant subi une montée en température de 25 à $90^{\circ} \mathrm{C}$ dans le four à micro-ondes.

Evolution of the compression stress as a function of the compression ratio $(\Delta \mathrm{h} / \mathrm{ho})$ and of the $\mathrm{pH}$, of whey protein concentrate at $10 \%$ protein heated from 25 to $90^{\circ} \mathrm{C}$ in a micro-wave oven.

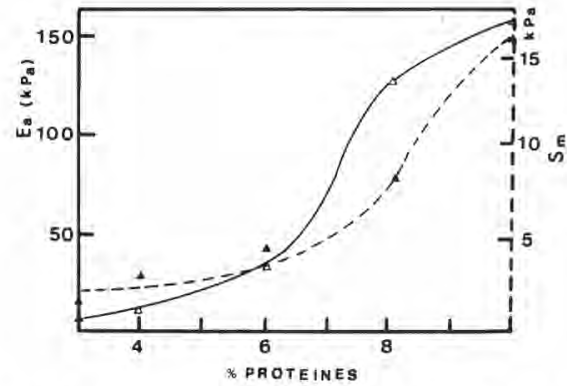

Fig 9. Évolution de la contrainte $\left(S_{m}\right)$ à $22 \%$ de taux de compression (ligne pointillée) et du module d'Young apparent $\left(E_{a}\right)$ à $2 \%$ de taux de compression (ligne continue) en fonction de la teneur en protéines. Les gels ont été obtenus à partir de solutions protéiques à $\mathrm{pH} 7$, ayant subi une montée en température de 25 à $90^{\circ} \mathrm{C}$ dans le four à micro-ondes, puis chauffés $30 \mathrm{~min}$ dans un bain d'eau à $90^{\circ} \mathrm{C}$.

Evolution of the maximal stress $\left(\mathrm{S}_{\mathrm{m}}\right)$ at the compression ratio of $22 \%$ (broken line) and of the apparent Young's modulus $\left(E_{a}\right)$ at the compression ratio of $2 \%$ (continuous line) as a function of protein concentration. Gels have been prepared from the protein solutions at $\mathrm{pH} 7 \mathrm{heated}$ from 25 to $90^{\circ} \mathrm{C}$ in a micro-wave oven and then kept for $30 \mathrm{~min}$ in a water bath at $90^{\circ} \mathrm{C}$.

entre 6 et $8 \%$ de protéines (respectivement 290 et $90 \%$ ); entre 8 et $10 \%$, elle devient plus faible pour $E_{a}(25 \%)$ et stagne pour $S_{m}(90 \%)$.

La figure 10 montre l'évolution de $E_{a}$ et de $S_{m}$ de gels obtenus à partir de solutions à $10 \%$ de protéines et à $\mathrm{pH} 7$, ayant subi une montée en température de 25 à $80^{\circ} \mathrm{C}$ (gel 1) ou $90{ }^{\circ} \mathrm{C}$ (gel 2) dans le four à micro-ondes. Le traitement thermique était ensuite poursuivi dans un bain-marie à 2 temps de chauffage (10 et $30 \mathrm{~min}$ ). Pour une même durée de chauffage, $E_{a}$ et $S_{m}$ des gels augmentent avec la température. Plus le temps de chauffage est élevé, plus l'écart entre les gels 1 et 2 se réduit : à $t=$ 0 dans le bain d'eau, $E_{a}$ est 4 fois plus élevé à $90^{\circ} \mathrm{C}$ qu'à $80^{\circ} \mathrm{C}, 1,3$ fois à $t=10$ 


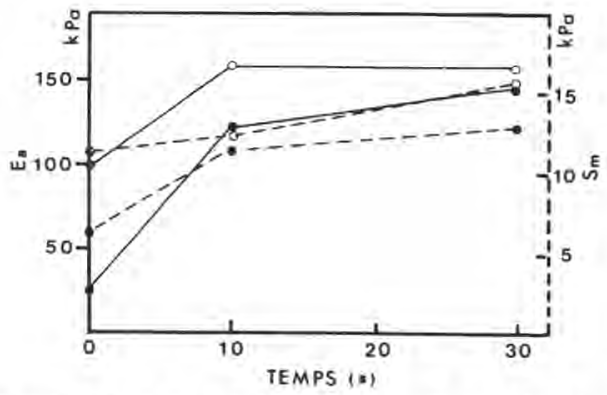

Fig 10. Evolution de la contrainte $\left(S_{m}\right)$ à $22 \%$ de taux de compression (lignes pointillées) et du module d'Young apparent $\left(E_{a}\right)$ à $2 \%$ de taux de compression (lignes continues) en fonction du temps de chauffage dans le bain d'eau. Les gels ont été obtenus à partir d'une solution à $10 \%$ de protéines et à pH 7 ayant subi un chauffage de 25 à 80 ou $90^{\circ} \mathrm{C}$ dans le four à micro-ondes, prolongé au bain d'eau à $80^{\circ} \mathrm{C}(-)$ ou $90^{\circ} \mathrm{C}$ (O-O) pendant 10 ou $30 \mathrm{~min}$.

Evolution of the maximal stress $\left(S_{m}\right)$ at the compression ratio of $22 \%$ (broken lines) and of the apparent Young's modulus $\left(E_{a}\right)$ at the compression ratio of $2 \%$ (continuous lines) as a function of the heating time. Gels have been prepared from the solutions containing $10 \%$ protein at $\mathrm{pH}$ 7 , heated from 25 to $80^{\circ} \mathrm{C}$ or $90^{\circ} \mathrm{C}$ in a microwave oven, and then kept for 10 or $30 \mathrm{~min}$ in a water bath at $80^{\circ} \mathrm{C}(-)$ or at $90^{\circ} \mathrm{C}(\mathrm{O}-\mathrm{O})$.

min et 1,1 fois à $t=30 \mathrm{~min}$; à $t=0$ et $t=30$ $\mathrm{min}, S_{m}$ augmente respectivement de 1,8 et de 1,2 fois entre ces 2 températures. A $80{ }^{\circ} \mathrm{C}$, l'augmentation de la durée de chauffage entraîne une augmentation de $E_{a}$ de $480 \%$ entre $0 \mathrm{~min}$ et $10 \mathrm{~min}$, et de $20 \%$ entre $10 \mathrm{~min}$ et $30 \mathrm{~min}$; à $90^{\circ} \mathrm{C}$, l'augmentation, moins importante, est respectivement de $60 \%$ et de $0 \%$. L'augmentation de $S_{m}$ est respectivement de $82 \%$ et de $16 \%$ à $80{ }^{\circ} \mathrm{C}$, de $9 \%$ et de $28 \%$ à $90{ }^{\circ} \mathrm{C}$. L'effet dénaturant de la température sur les protéines n'est pas le même à $80^{\circ} \mathrm{C}$ et à $90^{\circ} \mathrm{C}$, ce qui se traduit par une organisation et des caractéristiques rhéologiques différentes des gels 1 et 2 . L'effet plus pro- noncé de la durée de chauffage sur les caractéristiques mécaniques des gels 1 peut s'expliquer par l'existence de valeurs maximales vers lesquelles tendent $E_{a}$ et $S_{m}$, traduisant alors une limite à l'évolution physique des gels de CPL, plus rapidement atteinte pour les gels 2. Aux 2 températures étudiées, seule la solution à $\mathrm{pH} 3$ ne gélifie pas. Nous avons cependant obtenu un gel après une montée en température de 25 ${ }^{\circ} \mathrm{C}$ à $100{ }^{\circ} \mathrm{C}$ au four à micro-ondes (soit $160 \mathrm{~s}$ de chauffage). L'importance des forces répulsives intermoléculaires à ce $\mathrm{pH}$ est certainement à l'origine de ce résultat. La diafiltration du CPL, en contribuant à l'augmentation des forces répulsives, est donc néfaste à la gélification à $\mathrm{pH}$ très acide, comme l'ont déjà observé Dunkerley et Zadow (1981).

La figure 11 montre l'évolution de $E_{a}$ et de $S_{m}$ de gels obtenus à partir d'une solution à $10 \%$ de protéines ayant subi une montée en température de 25 à $90^{\circ} \mathrm{C}$ dans le four à micro-ondes. $E_{a}$ est le plus élevé à $\mathrm{pH} 4$ et 10 et le plus faible à $\mathrm{pH} 5$ et 11 : il est respectivement de 191 et 154 d'une part, de 12 et de $24 \mathrm{kPa}$ d'autre part. A $\mathrm{pH}$ voisin, le module d'Young apparent du gel de blanc d'œuf ( $\mathrm{pH} 8,25$ ) est environ 2 fois plus élevé que celui du gel de séroprotéines obtenu à pH 9 (143 contre $74 \mathrm{kPa})$. La contrainte à $22 \%$ de taux de compression est la plus élevée à $\mathrm{pH} 10$ (> $18 \mathrm{kPa}$ ) et $8(16 \mathrm{kPa})$ et la plus faible à $\mathrm{pH}$ $5(3 \mathrm{kPa})$ et $4(7 \mathrm{kPa})$. Entre $\mathrm{pH} 5$ et 8 , nous observons une augmentation de $S_{m}$, puis une baisse à pH 9 .

Les grandeurs $E_{a}$ et $S_{m}$ sont fréquemment utilisées pour décrire respectivement l'élasticité et la fermeté du produit (Prentice, 1987). L'élévation du pH entre 5 et 10 entraîne une augmentation de la fermeté et de la résistance à la perte d'eau par compression des gels obtenus à $90^{\circ} \mathrm{C}$, liée probablement à l'augmentation de l'hydratation des protéines, ce qui d'une part 


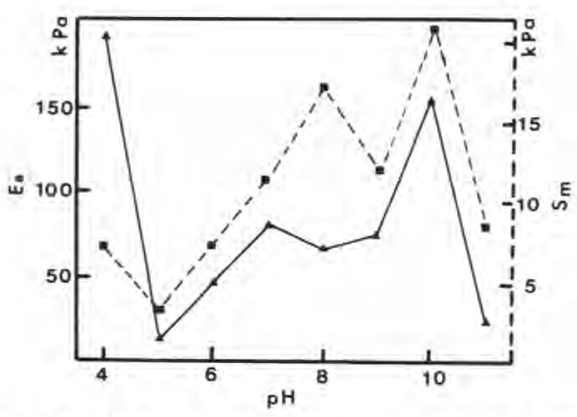

Fig 11. Évolution de la contrainte $\left(\mathrm{S}_{\mathrm{m}}\right)$ à $22 \%$ de taux de compression (ligne pointillée) et du module d'Young apparent $\left(E_{a}\right)$ à $2 \%$ de taux de compression (ligne continue) en fonction du pH. Les gels ont été obtenus à partir d'un concentré à $10 \%$ de protéines ayant subi une montée en température de 25 à $90^{\circ} \mathrm{C}$ dans le four à microondes.

Evolution of the maximal stress $\left(S_{m}\right)$ at the compression ratio of $22 \%$ (broken line) and of the apparent Young's modulus ( $\left.E_{a}\right)$ at the compression ratio of $2 \%$ (continuous line) as a function of $\mathrm{pH}$. Gels have been prepared from the solutions containing $10 \%$ protein heated from 25 to 80 or $90^{\circ} \mathrm{C}$ in a micro-wave oven.

se traduit par une diminution de la quantité d'eau libre et d'autre part par une augmentation du volume hydrodynamique des protéines. La présence d'ions positifs à $\mathrm{pH}$ alcalin revêt donc une grande importance lors de la gélification thermique de CPL puisque ceux-ci ont plus d'affinité que l'eau pour les sites hydrophiles des protéines. Ces résultats sont à rapprocher de l'étude de Dunkerley et Zadow (1981) qui ont travaillé sur un concentré protéique provenant d'un lactosérum acide : le gel présentait une fermeté moyenne à $\mathrm{pH} 5$ et excellente à $\mathrm{pH}>8$. Zirbel et Kinsella (1988) ont également observé à $70^{\circ} \mathrm{C}$ une augmentation de la fermeté des gels avec l'élévation de $\mathrm{pH}$ entre 6 et 8 , mais une diminution lorsque la température de chauffage était de 80 ou $90^{\circ} \mathrm{C}$. La concentration protéique étudiée par ces auteurs était cependant de $20 \%$ et le volume de la solu- tion traitée thermiquement de $1 \mathrm{ml}$ (soit 100 fois moins que le volume que nous avons mis en œuvre). A pH identique (89 ) et pour une concentration en protéines de $10 \%$, le gel obtenu à partir du blanc d'œuf est plus ferme et plus élastique que le gel de CPL. Selon Hermansson et Akesson (1975a et b), les gels d'ovalbumine et même de protéines de soja sont plus fermes, plus élastiques, plus résistants à la perte d'eau par compression que les gels de séroprotéines.

La combinaison des 2 paramètres, module d'Young apparent et contrainte de compression maximale, permet de rendre compte de la fragilité des gels. Ainsi, une forte valeur de $E_{a}$ pour une faible valeur de $S_{m}$ témoigne de la fragilité du gel, tandis que l'inverse reflète une structure moins cassante. Une démarche semblable a été effectuée par Kimura et al (1973) afin de caractériser la friabilité de gels alimentaires à partir du module d'Young et de la contrainte à la rupture. Nous proposons d'appeler «indice de fragilité", noté $I F$, le rapport $E_{a} / S_{m}$. C'est à $\mathrm{pH} 4$ que le gel de séroprotéines est le plus cassant (IF $=27$ ) et à $\mathrm{pH} 11$ le moins fragile (IF = 2,7). L'IF est de $3,8,3,8,6,1,6,2$ et 6,8 respectivement pour les gels obtenus à pH 5, 8, 9, 6 et 7. II est très intéressant de constater que ces résultats concordent globablement avec ceux de la description sensorielle des gels.

\section{Caractérisation sensorielle}

Avant chauffage et au $\mathrm{pH}$ d'origine de la solution ( $\mathrm{pH} 6,5)$, la solution est marron. Aux $\mathrm{pH}$ extrêmes, la couleur orangemarron domine et à $\mathrm{pH} 5$, un très léger trouble blanchâtre apparaît. L'aspect des gels obtenus à partir d'une solution protéique à $10 \%$, à différents $\mathrm{pH}$ (3 à 10) et ayant subi une montée en température de 
25 à $90^{\circ} \mathrm{C}$ dans le four à micro-ondes, est indiqué dans le tableau II. L'appréciation visuelle et tactile a été réalisée à partir de 7 descripteurs qualitatifs ou quantitatifs. Dans ce dernier cas, une note de 1 à 5 a été attribuée au gel : par exemple, lorsque la note de fragilité du gel est de 5 , cela signifie que le gel est très cassant alors qu'à 1 , il ne l'est pas. Entre pH 5 et 9 , le gel blanc et opaque présente une synérèse, ainsi qu'un effritement d'autant plus importants que le $\mathrm{pH}$ est bas. De plus, la cassure devient moins franche. La dénomination de gel s'applique plus à pH 9 qu'à pH 5 , où il s'agit plutôt d'un précipité compte tenu de la présence d'une fine pellicule d'un liquide clair à la surface du gel. Nous observons qu'aux pH extrêmes étudiés (4, 10 et 11), les gels présentent un aspect similaire : ils sont translucides, ne s'effritent pas et emprisonnent bien l'eau. Ces points communs n'en cachent pas moins une grande différence : les gels à pH 10 et 11 sont moins fragiles et présentent une cassure nettement plus franche qu'à pH 4. Les caractéristiques sensorielles du gel de blanc d'œuf se rapprochent de celles du gel de CPL obtenu à $\mathrm{pH} 9$, avec cependant un effritement plus faible, une plus grande fermeté et enfin une saveur typique à ce produit. Les gels obtenus aux concentrations inférieures à $10 \%$ de protéines sont moins fermes, plus cassants et présentent une synérèse accrue quel que soit le $\mathrm{pH}$ étudié. $\mathrm{A} \mathrm{pH} 4$ et 10 , le gel est plus translucide; de $\mathrm{pH} 5$ à 9 , il demeurait blanc et opaque.

II est très important de souligner qu'à partir de la simple et unique variation de $\mathrm{pH}$, il est possible d'obtenir toute une gamme de texture de gels de séroprotéines. Bien que non abordé dans cette étude, l'environnement minéral joue un rôle déterminant dans la texture des gels thermiques de CPL. Harwalkar et Kalab (1985) ont observé en microscopie électronique une fine structure des gels en présence de $200 \mathrm{mmol} / \mathrm{l}$ de $\mathrm{NaCl}$ ( $9 \%$ protéines, $\mathrm{pH} 2,5,30 \mathrm{~min}$ à $90^{\circ} \mathrm{C}$ ). A 400 $\mathrm{mmol} / \mathrm{l}$ de $\mathrm{NaCl}$, la structure du gel est très agrégée et la taille des agrégats constituant le gel augmente. Par la même technique, Mulvihill et Kinsella (1988) ont observé un phénomène semblable sur des gels

Tableau II. Évaluation sensorielle de gels obtenus à différents $\mathrm{pH}$ à partir des solutions à $10 \%$ de protéines ayant subi une montée en température de 25 à $90^{\circ} \mathrm{C}$ au four à micro-ondes.

Odeur : FO = Flanc d'œuf, LP = légèrement piquante; Couleur : $\mathrm{OM}=$ Orange-Marron.

Sensory evaluation of gels prepared from the solutions containing $10 \%$ proteins at different $\mathrm{pH}, \mathrm{by}$ heating from 25 to $90^{\circ} \mathrm{C}$ in a micro-wave oven.

\begin{tabular}{llcccccccc}
\hline \multicolumn{1}{c}{$p H$} & 4 & 5 & 6 & 7 & 8 & 9 & 10 & 11 \\
\hline Odeur & Odor & - & FO & FO & FO & FO & FO & - & LP \\
Couleur & Color & blanc & blanc & blanc & blanc & blanc & blanc & blanc & OM \\
Opacité & Opacity & 2 & 5 & 5 & 5 & 5 & 5 & 2 & 1 \\
Effritement & Crumbling & 1 & 5 & 4 & 3 & 2 & 1 & 1 & 1 \\
Fermeté & Firmness & 1 & 1 & 2 & 3 & 4 & 4 & 5 & 3 \\
Synérèse & Syneresis & 1 & 5 & 4 & 3 & 2 & 1 & 1 & 1 \\
Fragilité & Fragility & 5 & 3 & 2 & 2 & 3 & 3 & 2 & 1 \\
\hline
\end{tabular}


de $\beta$-lactoglobuline $(10 \%, \mathrm{pH} 8,30 \mathrm{~min}$ à $90^{\circ} \mathrm{C}$ ) entre 5 et $15 \mathrm{mmol} / / \mathrm{de} \mathrm{CaCl}_{2}$. La composition en calcium et en sodium du CPL étudié est respectivement de 180 et $80 \mathrm{mmol} / \mathrm{l}$ pour $10 \%$ de protéines solubles. La concentration très élevée en calcium explique très certainement le caractère agrégé des gels obtenus entre $\mathrm{pH} 5$ et 9 , déjà observé par Hillier et al (1980). La couleur blanche opaque de ces gels conduit à penser que les protéines s'insolubilisent avant de former un gel. La fixation du calcium sur les groupements carboxyliques chargés négativement permet la mise en place de ponts calciques intermoléculaires, entraînant dans un premier temps la formation d'agrégats de protéines. C'est d'ailleurs cette hypothèse qu'émettent Zittle et Dellamonica (1956) à propos de la $\beta$-lactoglobuline, De Wit (1981) et Mulvihill et Kinsella (1988) à propos de concentré de lactosérum pour expliquer des phénomènes de floculation survenant en présence de calcium. Dans un second temps, il s'établirait des liaisons entre ces agrégats, entraînant la formation d'un réseau protéique.

Les résultats obtenus lors de ces travaux nous ont conduit à nous interroger quant à la participation à ce phénomène des différentes protéines présentes dans le lactosérum. Nous avons vu le concentré protéique présentait une bonne aptitude à la gélification à $\mathrm{pH} 4$. Ceci montre que la $\beta$-lactoglobuline n'est pas la seule protéine à intervenir dans le phénomène de gélification, car selon Gault (1987) et Paulsson et al (1986), cette protéine ne gélifie pas à $\mathrm{pH}$ acide en solution individuelle. Les autres protéines interviennent également, notamment la sérum albumine $(4 \%$ des protéines sériques) qui présente, seule ou en mélange avec les autres séroprotéines, une très bonne aptitude à la gélification à
$\mathrm{pH}$ acide (Paulsson et al, 1986). Bien que possédant de meilleures propriétés d'agrégation que la $\beta$-lactoglobuline à faible concentration $(<5 \mathrm{~g} / \mathrm{l})$, à $\mathrm{pH} 4,2$ et sous l'effet d'un traitement thermique modéré de $65^{\circ} \mathrm{C}$ pendant 30 min (Pearce, 1983), l' $\alpha$ lactalbumine ( $20 \%$ des protéines sériques) a un très faible pouvoir gélifiant $(<200 \mathrm{~g} /$; 30 à $\left.95^{\circ} \mathrm{C}, 1^{\circ} \mathrm{C} / \mathrm{min}\right)$, très certainement en raison de l'absence de groupements SH libre (Gault, 1987; Paulsson et al, 1986). En outre, Paulsson et al (1986) ont montré que cette protéine a un effet inhibiteur sur la gélification des autres protéines sériques. La gélification résulte en fait d'un effet synergique entre les différentes protéines présentes dans la solution. II serait d'ailleurs intéressant d'établir les concentrations nécessaires de chacune de ces protéines afin d'optimiser la gélification thermique du CPL.

De nombreux travaux ont été réalisés sur l'influence d'un paramètre donné sur la gélification thermique des protéines sériques, mais peu sur l'interaction de plusieurs facteurs. Or, nous avons montré que la concentration minimale en protéines et la température de début de gélification sont très dépendantes du $\mathrm{pH}$. C'est dans cette voie qu'il faut poursuivre ces travaux, c'est-à-dire dans l'interdépendance qui existe entre les différents facteurs $(\mathrm{pH}, \mathrm{mi}-$ néraux, lipides, lactose, concentration optimale de chacune des protéines sériques), afin de mieux comprendre ce phénomène complexe qu'est la gélification thermique du concentré de séroprotéines.

\section{REMERCIEMENTS}

Nous remercions $\mathrm{JL}$ Maubois pour l'aide qu'il nous a apportée dans la rédaction de cette publication. 


\section{RÉFÉRENCES}

Acton GH (1977) Determination of lactose in cheese. Aust J Dairy Techno/ 32, 111

Andrade EN (1930) The viscosity of liquids. Nature 125, 309-310

Bingham EC, Jackson MH (1918) Viscosity and fluidity of water. In: Handbook of chemistry and physics (CD Hodgman, ed). The chemical rubber publishing co, 2257

Brulé G, Maubois JL, Fauquant J (1974) Étude de la teneur en éléments minéraux des produits obtenus lors de l'ultrafiltration du lait sur membrane. Lait 54, 600-615

Burgess KJ, Kelly J (1979) Technical note: selected functional properties of a whey protein isolate. J Food Technol 14, 325-329

Cardenas-Caroti R, Grosclaude G, Nicol G, Maubois JL (1985) Rhéomètre pour l'étude de produits conformes en corps non linéaires. Brevet français $n^{\circ} 2588379$

Cooper HR, Hugues JR, Matthews ME (1977) Application of "response surface methodology" to the evaluation of whey protein gel systems. N Z J Dairy Sci Technol 12, 248-252

De Wit JN, De Boer R (1975) Ultrafiltration of cheese whey and some functional properties of the resulting whey protein concentrate. Neth Milk Dairy J 29, 198-211

De Wit JN (1981) Structure and functional behaviour of whey proteins. Neth Milk Dairy J 35, 47-64

De Wit JN, Klarenbeek G (1984) Effects of various heat treatments on strucfure and solubility of whey proteins. $J$ Dairy Sci 67,2701 2710

Dunkerley JA, Hayes JF (1980) Characterization of whey protein gels using a temperature gradient block. N Z J Dairy Sci Technol 15, 191196

Dunkerley JA, Zadow JG (1981) Rheological studies on heat induced coagula from whey protein concentrates. N Z J Dairy Sci Technol $16,243-252$

Fauquant J, Pierre A, Brulé G (1985a) Clarification du lactosérum acide de caséinerie. Tech Lait 1003, 37-41

Fauquant $\mathrm{J}$, Vieco $\mathrm{E}$, Brulé $\mathrm{G}$, Maubois $\mathrm{JL}$ (1985b) Clarification des lactosérum doux par agrégation thermocalcique de la matière grasse résiduelle. Lait 65, 1-20

FIL (1959) Détermination de la matière grasse dans le fromage et le fromage fondu par la méthode de Schmid-Bondzynski-Ratzlaff. Int Stand FIL-IDF 5

Gault $P$ (1987) Influence du traitement thermique et mécanique sur le comportement rhéologique des protéines solubles du lait et conséquences sur la viscosité apparente des fromages frais enrichis en protéines solubles. Mémoire, école supérieure d'agriculture d'Angers

Hagett TOR (1976) The whipping, foaming and gelling properties of whey protein concentrates. N Z J Dairy Sci Technol 11, 244-250

Harwalkar VR, Kalab M (1985) Thermal denaturation and aggregation of $\beta$-lactoglobulin in solution. Electron microscopic study. Milchwissenschaft 40, 65-68

Hermansson AM, Akesson C (1975a) Functional properties of added proteins correlated with properties of meat systems: effect of concentration on water-binding properties of model meat systems. J Food Sci 40, 595-602

Hermansson AM, Akesson C (1975b) Functional properties of added proteins correlated with properties of meat systems: effect of salt on water-binding properties of model meat system. J Food Sci 40, 603-610

Hillier RM, Cheeseman GC (1979) Effect of proteose-peptone on the heat gelation of whey protein isolate. J Dairy Res 46, 113-120

Hillier RM, Lyster RLJ, Cheeseman GC (1980) Gelation of reconstituted whey powders by heat. J Sci Food Agric 31, 1152-1157

Johns JEM, Ennis BM (1981) The effects of the replacement of calcium with sodium ions in acid whey on the functional properties of whey protein concentrates. $N Z$ Dairy $S c i$ Technol 16, 79-86

Kimura H, Moritaka S, Misaki M (1973) Polysaccharide 13140: A new thermo-gelable polysaccharide. J Food Sci 38, 668-670

Kinsella JE (1984) Milk proteins: physicochemical and functional properties. CRC Crit Rev Food Sci Nutr 21, 197-262

Korolczuk J, Roignant M, Maubois JL (1985) Computer data acquisition of sinusoidally varying stress and strain. J Texture Stud 16, 129-142 
Lee CH, Rha C (1979) Rheological properties of proteins in solution. In: Food texture and rheology (P Sherman, ed) Academic Press, London, 245-263

McDonough FE, Hargrove RE, Mattingly WA, Posati LP, Alford JA (1974) Composition and properties of whey protein concentrates from ultrafiltration. J Dairy Sci 57, 1438-1443

Morr CV (1979) Functionality of whey protein products. Review. N Z J Dairy Sci Technol 14, 185-194

Morr CV, German B, Kinsella JE, Regenstein JM, Van Buren JP, Kilara A, Lewis BA, Mangino ME (1985) A collaborative study to develop a standardized food protein solubility procedure. J Food Sci 50, 1715-1718

Mulvihill DM, Kinsella JE (1987) Gelation characteristics of whey proteins and $\beta$ lactoglobulin. Food Technol 41, 102-111

Mulvihill DM, Kinsella JE (1988) Gelation of $\beta$ lactoglobulin: effects of sodium chloride and calcium chloride on the rheological and structural properties of gels. J Food Sci 53, 231236

Paulsson M, Hegg PO, Castberg HB (1986) Heat-induced gelation of individual whey proteins. A dynamic rheological study. $J$ Food Sci $51,87-90$
Pearce RJ (1983) Thermal separation of $\beta$ lactoglobulin and $\alpha$-lactalbumin in bovine cheddar cheese whey. Aust $J$ Dairy Technol 38, 144-149

Prentice JH (1987) Cheese rheology. In: Cheese: chemistry, physics and microbiology (PF Fox, ed), Elsevier Applied Science, 299344

Rowland SJ (1938) The determination of nitrogen distribution in milk. J Dairy Res 9, 42-46

Serres L, Amariglio S, Petransxiene D (1973) Contrôle de la qualité des produits laitiers. Direction des services vétérinaires

Sternberg M, Chiang JP, Eberts NJ (1976) Cheese whey proteins isolated with polyacrylic acid. J Dairy Sci 59, 1042-1050

Wayne JEB, Shoemaker CF (1988) Rheological characterization of commercially processed fluid milks. J Texture Stud 19, 143-152

Zirbel F, Kinsella JE (1988) Factors affecting the rheological properties of gels made from whey protein isolates. Milchwissenschaft 43, 691-694

Zittle CA, Dellamonica ES (1956) Viscosity and flocculation of heated $\beta$-lactoglobulin solutions. J Dairy Sci 39, 514-521 